\title{
Interplay between Intrinsic Plasma Rotation and Magnetic Island Evolution in Disruptive Discharges ${ }^{1}$
}

\author{
G. Ronchi ${ }^{a}$, J. H. F. Severo ${ }^{a}$, F. Salzedas ${ }^{b, c}$, R. M. O. Galvão ${ }^{a}$, and E. K. Sanada ${ }^{a}$ \\ ${ }^{a}$ Instituto de Física, Universidade de São Paulo, São Paulo, Brazil \\ ${ }^{b}$ Universidade do Porto, Faculdade de Engenharia, Porto, Portugal \\ ${ }^{c}$ Instituto de Plasmas e Fusão Nuclear, Instituto Superior Técnico, Universidade de Lisboa, Lisboa, Portugal \\ e-mail:rgalvao@if.usp.br
}

Received September 24, 2015

\begin{abstract}
The behavior of the intrinsic toroidal rotation of the plasma column during the growth and eventual saturation of $m / n=2 / 1$ magnetic islands, triggered by programmed density rise, has been carefully investigated in disruptive discharges in TCABR. The results show that, as the island starts to grow and rotate at a speed larger than that of the plasma column, the angular frequency of the intrinsic toroidal rotation increases and that of the island decreases, following the expectation of synchronization. As the island saturates at a large size, just before a major disruption, the angular speed of the intrinsic rotation decreases quite rapidly, even though the island keeps still rotating at a reduced speed. This decrease of the toroidal rotation is quite reproducible and can be considered as an indicative of disruption.
\end{abstract}

DOI: $10.1134 / \mathrm{S} 1063780 \mathrm{X} 16050159$

\section{INTRODUCTION}

Magnetic islands play a crucial role in the dynamics of magnetically confined plasmas; they not only can drastically spoil the confinement of particles and energy, but, if their saturated size becomes a substantial fraction of the minor radius of the plasma column, they can lead to a major disruption of the discharge, with rather deleterious effects for the magnetic confinement device [1, 2]. For this reason, the dynamics of magnetic islands, under different experimental conditions, has been continuously and intensively investigated, since the pioneering works on resistive instabilities [3, 4] and their nonlinear evolution [5-7].

Two associated effects were found to play a crucial role on the temporal evolution of magnetic islands, rotation and interaction with the finite resistive walls of the vacuum chamber or with external error fields [8, 9]. The rotation of the perturbed fields associated with magnetic islands is basically driven by equilibrium gradients, i.e., drift effects, so that the rotation is observed, even in a static plasma column, at frequencies close to the electron diamagnetic frequency [4, 7]. However, due to viscosity, interaction with external error fields, and/or dissipation at the external resistive wall, the rotation may substantially slow down, leading to the so-called locked modes that can trigger major plasma disruptions $[2,9,10]$.

\footnotetext{
${ }^{1}$ The article is published in the original.
}

In previous experiments carried out in TCABR, it was found that the measured values for plasma poloidal rotation velocity agreed, within the error limits, with the neoclassical predictions, almost everywhere over the plasma cross section [11]. The values for the toroidal velocity, on the other hand, although not quite in agreement with theoretical predictions, agreed with experimental results obtained in similar tokamaks. However, at the radial positions corresponding to the mode rational surfaces $q=2$ and $q=3$, it was found that the values of these velocities were somewhat larger and this result was interpreted as the plasma locally rotating locked to the magnetic islands [12]. However, in these experiments accurate determination of the temporal evolution of the plasma rotation was not available, making impossible a close verification of this result as the islands evolved.

Due to their relevance for disruption scenarios in large future tokamaks, in which the plasma rotation is expected to be small, the evolution and the external control of magnetic islands in rotating plasma columns has been extensively studied, both theoretically and experimentally, for over two decades [10, 13-15]. In these studies, the main concern was to establish a threshold for error fields to excite magnetic islands and to investigate their control with external, static or dynamic, resonant coils, or with active modification of the relevant plasma parameters inside the island, such as temperature and current density. 
In the majority of the experiments carried out so far, the plasma rotation was driven by external momentum input, usually through controlled neutral beam injection (NBI) [15]. It turns out, however, that the plasma column in tokamaks has a so-called spontaneous or intrinsic rotation, which can be driven by different mechanisms [16-18]. Although the intrinsic angular velocity is usually smaller than that of the island, it may play an important role on the island evolution, in situations where NBI is not available, or not effective, to drive angular momentum.

Since this interaction has not been systematically investigated yet, we carried out a detailed study of the evolution of both the island and intrinsic rotation in disruptive discharges in TCABR, in which the disruption is triggered by a programmed density rise. This triggers the tearing instability and the resulting magnetic island initially rotates at angular velocity much higher than that of the plasma. As the island grows, it slows down, as predicted by theory [9, 10, 19], and the plasma rotation increases, with the angular velocities approaching each other. However, when the island reaches saturation at a large size, and just before a major disruption, the angular velocity of the spontaneous rotation decreases rapidly toward zero, even though the island keeps still rotating at a reduced frequency.

\section{EXPERIMENTAL SETUP}

TCABR is a small size tokamak, with the following main parameters: the major radius $R=0.61 \mathrm{~m}$, minor radius $a=0.18 \mathrm{~m}$, toroidal magnetic field $B_{T}=1.1 \mathrm{~T}$, plasma current $I_{p} \leq 100 \mathrm{kA}$, line-averaged electron plasma density $n_{e} \leq 4 \times 10^{19} \mathrm{~m}^{-3}$, and central electron

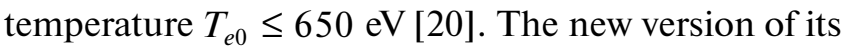
spectroscopic diagnostic system allows measuring the temporal evolution of the rotation of chosen impurities inside the plasma. Actually, passive spectroscopy has long been employed to measure the Doppler shift of impurity lines, caused by plasma rotation, in other experiments [21]. However, it is known that this method is quite susceptible to experimental errors, in particular regarding the measurement of the poloidal rotation velocity $[22,23]$, and does not provide the spatial resolution of the standard CXRS technique used in large tokamaks with NBI [24].

The main distinctive feature of the system installed in TCABR is that it uses an original scheme of splitting the spectral line at the output of the spectrometer. The integrated signals from the two halves are measured by independent detectors and these signals should be equal in the absence of Doppler shift. When there is a Doppler shift, the line displacement causes a difference between them, so that the Doppler velocity can be determined from their ratio. This scheme can be absolutely calibrated by measuring the poloidal rotation using a line-of-sight passing through the mag- netic axis, where it is supposed to vanish. Details of this diagnostic system and an error analysis of the measured signal can be found in [25]. With the present set up, the toroidal velocity can be measured with time resolution equivalent to $600 \mathrm{~Hz}$ and precision somewhat better than $5 \mathrm{~km} / \mathrm{s}$, large but still appropriate for measuring the intrinsic toroidal velocity without the uncertainties associated with external moment input, which is the drawback of the CXRS technique.

The main parameters of the reference discharge chosen for this experimental campaign are shown in Fig. 1; the working gas is hydrogen. The values of the maximum plasma density and current $\left(n_{e 0} \cong 2.8 \times 10^{19} \mathrm{~m}^{-3}, I_{p, \max } \cong 80 \mathrm{kA}\right.$, respectively) were chosen to avoid disruptions and to have low MHD activity (Fig. 1e). The central electron temperature, measured in the current flat top, is around $500 \mathrm{eV}$. The plasma density profile follows approximately the standard one for ohmic discharges in TCABR, $n(r) \approx n_{e 0}\left[1-(r / a)^{2}\right]^{0.8}$. In the presence of sawtooth oscillations, the soft $\mathrm{X}$-ray array (20 vertical channels) allows a good determination of the radial position of the $q=1$ rational magnetic surface. With this information, and taking into account that the plasma column of TCABR is circular $(q(r=a) \cong 3.5)$, we can estimate quite reasonably the position of the other rational magnetic surfaces. In particular, the position of the $q=2$ singular surface is around $r_{s}=$ $12 \mathrm{~cm}$. Considering the standard electron temperature profiles in TCABR, determined by electron cyclotron emission in low-density discharges, we estimate that the electron temperature in the $q=2$ surface to be between 80 and $100 \mathrm{eV}$, so that the electrons are in the transition between the Pfirsch-Schlüter and plateau regimes.

The poloidal and toroidal rotation velocities, both measured at $r \cong 8 \mathrm{~cm}$, somewhere between the $q=1$ and $q=2$ rational magnetic surfaces (Fig. 1h), are determined from the Doppler shift of the CVI impurity line. Therefore, to support the analysis of the behavior of the island rotation velocity in the sequel, it is important to discuss the relation between these velocities and the corresponding ones for the main hydrogen ions. Considering the predictions of neoclassical theory, we expect the poloidal velocity of the main ions to be quite different from that of the impurities, in particular close to the plasma edge, with the latter much larger than the former. The toroidal velocities of main ions and impurities, on the other hand, must be almost the same, in the collisional regime, "due to the strong parallel friction forces on parallel flows and weak viscous forces on poloidal flows" [26]. This is even more valid around the position of the $q=2$ rational surface. In fact, previous measurements of the impurity rotation at the plasma edge of TCBR have shown that the neoclassical prediction is reasonably verified experimentally [11, 12]. 

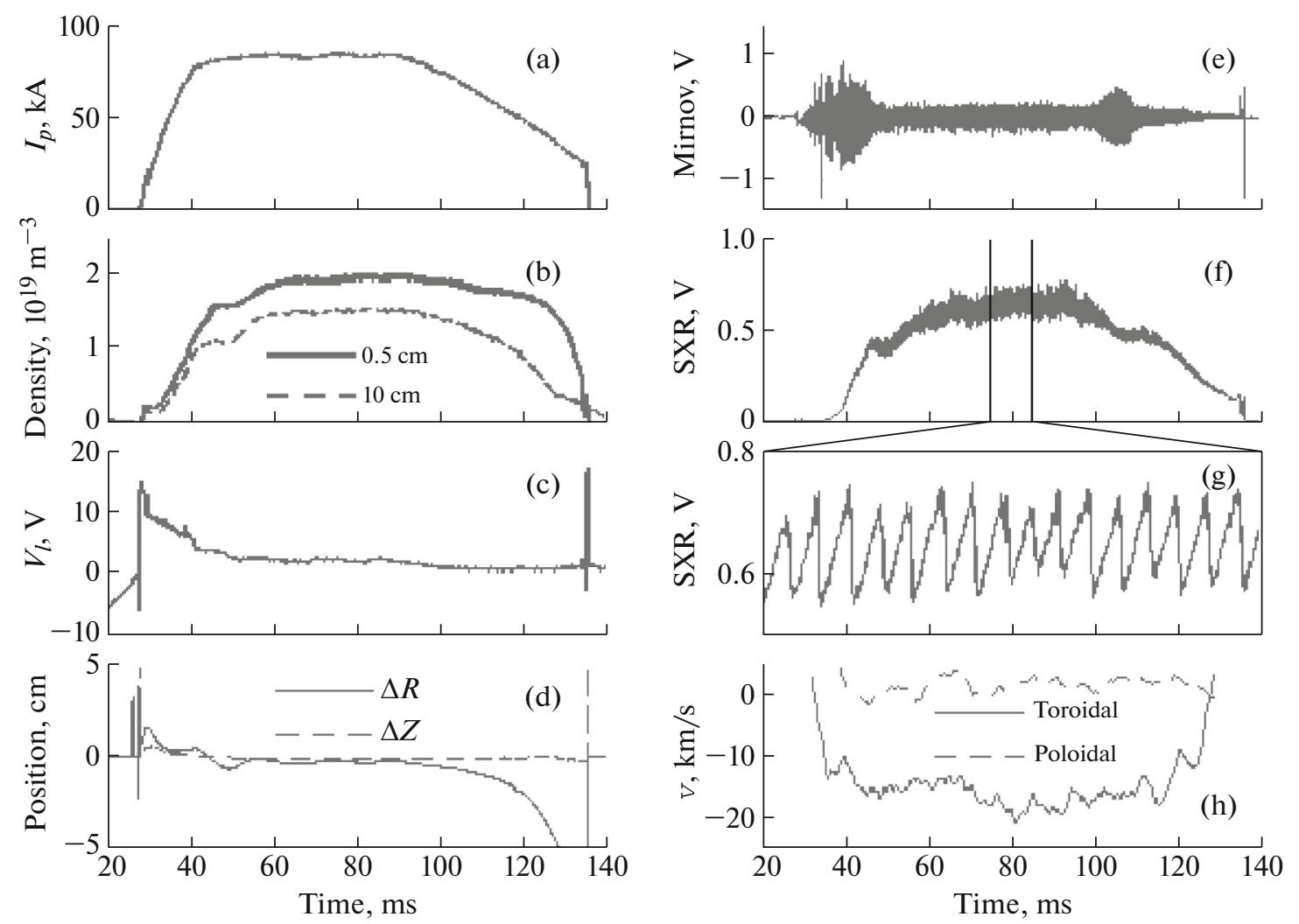

Fig. 1. Main parameters of the reference discharge no. 30457 of TCABR: (a) plasma current $I_{p}$, (b) line density $n_{e}$ at two viewing chords of the interferometer $(r=0.5 \mathrm{~cm}$ and $r=10 \mathrm{~cm})$, (c) loop voltage $V_{l}$, (d) radial $(\Delta r)$ and vertical $(\Delta z)$ displacements of the magnetic axis, (e) MHD signal, (f) central SXR emission, (g) blow-up of SXR emission, and (h) intrinsic toroidal and poloidal rotation velocities measured at $r=8 \mathrm{~cm}$ (for the toroidal velocity, positive means rotation in the direction of the plasma current and negative, in the direction of the toroidal magnetic field).

The intrinsic toroidal rotation is in the direction of the toroidal magnetic field and the poloidal one in the electron diamagnetic sense. TCABR operates with the plasma current opposite to the toroidal magnetic field, so that, looking in the current direction, the electron diamagnetic rotation is levorotatory. The intrinsic toroidal rotation increases during the current ramp up phase and keeps an average value around $15 \mathrm{~km} / \mathrm{s}$ during flat top, decreasing to zero at the end of the discharge. In standard discharges (with low MHD activity), the value of the intrinsic toroidal rotation decreases toward the plasma boundary and may even change the sense of rotation close to the edge $[12,25]$.

\section{DISRUPTIVE DISCHARGES}

In the experiments, a programmed density increase, starting around $t \approx 62 \mathrm{~ms}$, is employed to trigger the tearing mode and excite a large magnetic island, eventually leading to a disruption. The temporal behavior of the main plasma parameters in a typical discharge with density increase is shown in Fig. 2. The SXR signal indicates that, as the density increases, the plasma temperature drops somewhat and a strong $|m / n|=2 / 1$ MHD mode is destabilized, with initial angular velocity $\omega_{\mathrm{MHD}} \approx 9.4 \times 10^{4} \mathrm{~s}^{-1}$, where $m$ and $n$ are the poloidal and toroidal mode numbers, respectively (the Mirnov coils [24] in TCABR are placed around and close to the plasma column, inside two semicircular stainless steel tubes, so that the system allows quite good mode resolution [27]). The mode increases its amplitude smoothly and eventually saturates, just before a major plasma disruption occurs, starting around $t \approx 78 \mathrm{~ms}$. The discharge parameters were chosen such that practically only this mode was driven unstable, i.e., during its growing phase the other $m$ components had quite small amplitudes. However, in the saturation phase, $t>75 \mathrm{~ms}$, the magnetic signals show components with values of $m>2$ with amplitudes of the order of $12 \%$ of $m=2$.

The plasma intrinsic toroidal rotation at the magnetic island location is around $15 \mathrm{~km} / \mathrm{s}$ just before the mode excitation, which corresponds to an angular velocity $\omega_{T} \approx 2.4 \times 10^{4} \mathrm{~s}^{-1}$, therefore, about four times smaller than that of the mode. Naturally, since the viewing chord of the diagnostic system crosses the 

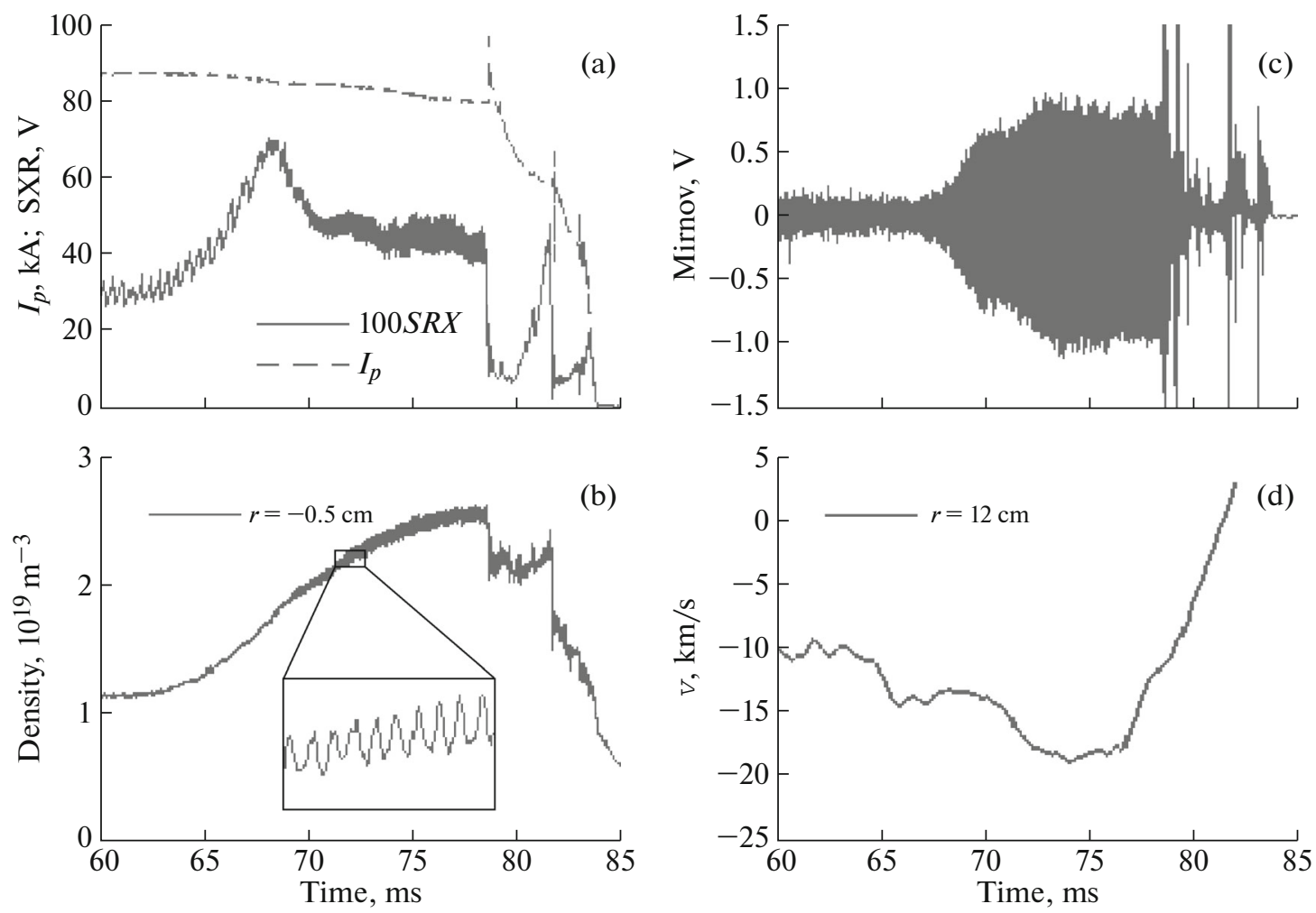

Fig. 2. (a) Plasma current and central SXR signal, (b) central electron density, (c) MHD activity, and (d) toroidal rotation velocity for typical discharge no. 30571 with density increase. The insert in panel (b) shows the density oscillations associated with density variations inside the rotating islands [28]. Their frequency match the one measured with the external Mirnov coils.

plasma column in the equatorial plane, tangent to the $q=2$ surface, the island passes periodically through it, so that the measured Doppler shift could be influenced by emission from impurities trapped in and moving with the island (we remark that the impurity transport across magnetic islands is still an open issue). However, considering that the time resolution of the spectroscopic diagnostic is equivalent to $600 \mathrm{~Hz}$, much smaller than the island frequency, this effect is somewhat averaged out. As the mode amplitude increases, the rotation frequency of the plasma increases and that of the mode decreases, so that $\Omega=\omega_{\mathrm{MHD}} / \omega_{T}$ approaches 2 , as will be discussed in the sequel. Actually, the intrinsic toroidal rotation of the plasma almost doubles, as shown in Fig. 2d. Furthermore, as the mode saturates and the disruption is approached, the intrinsic toroidal rotation decreases rapidly, tending to at least recover its value before the mode excitation. The rotation measurements after the disruption, at $t \approx 78 \mathrm{~ms}$, are not reliable because of the associated strong inward displacement of the plasma column, as can be inferred from the SXR signal in Fig. 2a.

To better investigate the behavior of the intrinsic toroidal rotation during the island growth and slowing down, we have measured it in similar discharges along lines-of-sight on both sides of the $q=2$ magnetic surface. The results are shown for three discharges, including 30571, in Fig. 3. In this figure

$$
w=4\left[\frac{q r R \tilde{b}}{m \tilde{s} B_{T}}\right]_{r=r_{s}}^{1 / 2}
$$

is the estimated island width [29], where $\tilde{s}$ and $\tilde{b}$ are the shear parameter and the island perturbed magnetic field, respectively, i.e.,

$$
\tilde{s}=\left(\frac{r}{q} \frac{d q}{d r}\right)_{r_{s}}, \quad \tilde{b}\left(r_{s}\right) \approx\left(\frac{0.12}{0.20}\right) \tilde{b}_{\text {Mirnov }} .
$$

In all discharges the island saturates at large amplitude, corresponding to about $1 / 3$ of the minor plasma radius (we note, however, that this is an upper limit calculated with the rather approximate theoretical expression given above). As the island grows, its frequency drops, quite fast in the beginning, somewhat slower when it is approaching saturation, and then fast again just before disruption (the measurements after disruption are not reliable). At the same time, the intrinsic toroidal rotation frequency increases up to almost half that of the island, at the mode rational surface. 

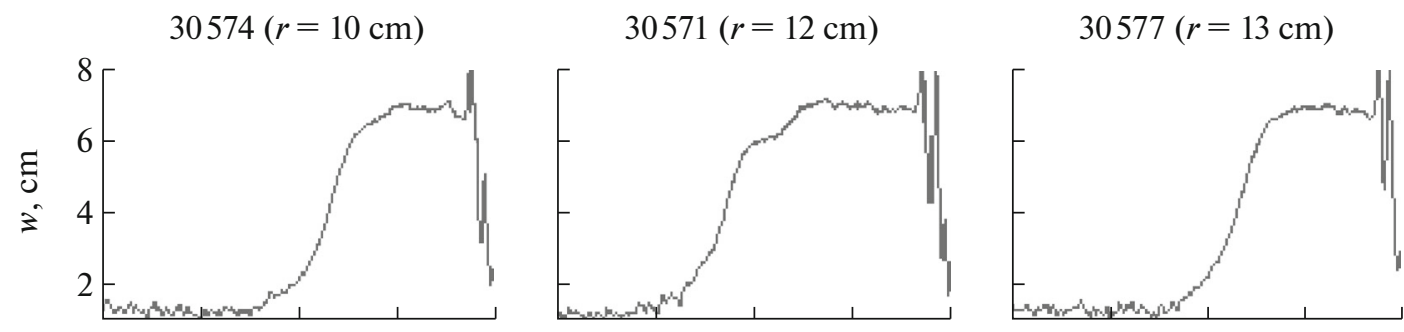

(a)
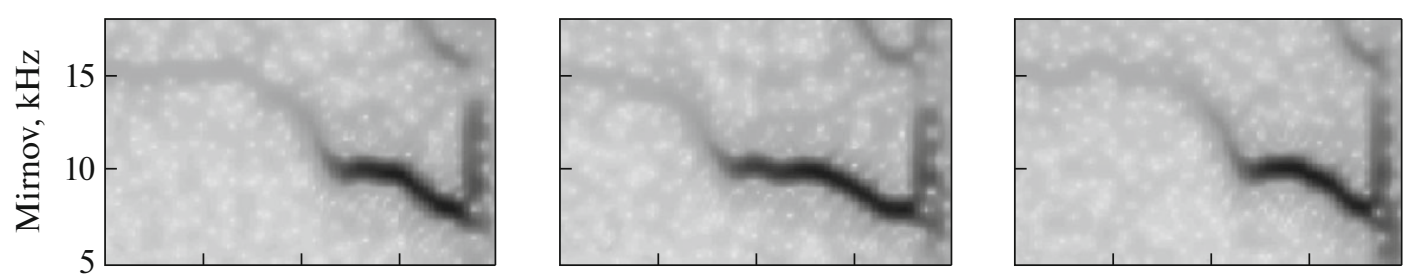

(b)
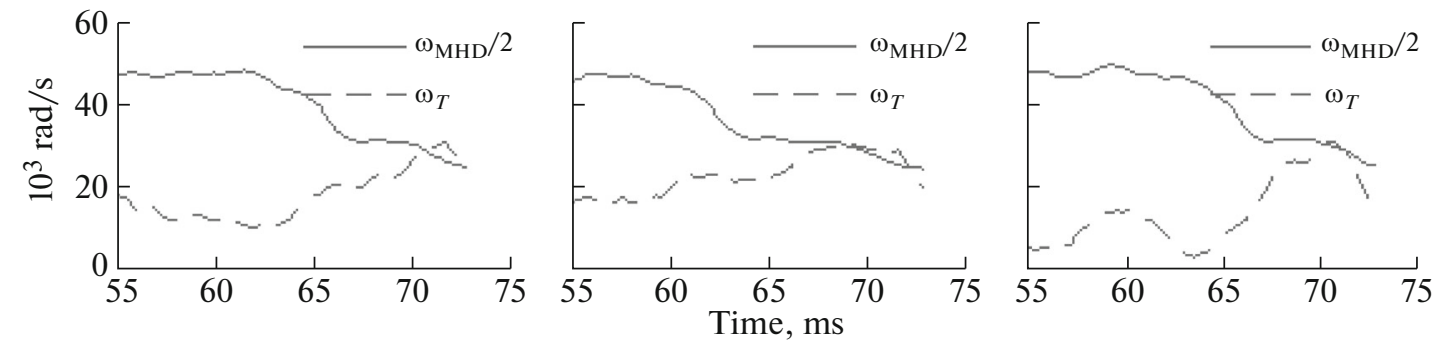

Fig. 3. (a) Island width; (b) spectrogram of Mirnov frequency; and (c) island, $\omega_{\mathrm{MHD}} / 2$, and plasma, $\omega_{T}$, angular velocities for three similar discharges, measured along different lines-of-sight in the toroidal direction and on the equatorial plane of the plasma column. The radius indicated for each discharge corresponds to the radius where the line-of-sight is tangent to the magnetic surface.

This behavior seems to be somewhat compatible with the simple theoretical prediction that, in absence of external torque, the island rotation should be damped, eventually locking to the rotation of the bulk plasma $[9,10]$ so that

$$
\omega_{\mathrm{MHD}}= \pm m \omega_{p}+n \omega_{T}+\omega_{\mathrm{drift}},
$$

where $\omega_{p}$ is the poloidal angular velocity of the plasma column and $\omega_{\text {drift }}$ is the "natural" angular frequency of the island, which is of the order of the electron diamagnetic angular velocity, i.e, $\omega_{*_{e}} \approx(m / r) V_{\text {dia }}$, where $V_{\text {dia }}$ is the diamagnetic velocity [10]. Unfortunately, due to practical assembly constraints, we have not directly measured the CVI poloidal angular rotation velocity at the position of the $q=2$ singular surface. However, we expect it to be small, in particular, in the phase when the island becomes large. From Fig. 1 and standard density and temperature profiles for TCABR, a maximum value can be estimated for the electron diamagnetic angular velocity when the island is still very small, $\omega_{*_{e}} \approx 17 \mathrm{krad} / \mathrm{s}$. As the island width increases and saturates, this value should decrease and become equal to the difference between the curves of $\omega_{T}$ and $\omega_{\text {MHD }}$. The fact these two curves do not converge when the island width saturates can be interpreted as an indication the pressure profile inside the island is not completely flat. Actually, previous measurements in TCABR have shown that not only the poloidal rotation varies with radius [11, 12], but also that the temperature profile is not flat across the $|m / n|=2 / 1$ islands [30]. Similar results have been obtained in other experiments; however ours presents a quite clear example for the case in which one can assure that there is no driven toroidal rotation, i.e., it is entirely intrinsic. In particular, we are not aware of other work reporting the increase of the intrinsic toroidal rotation as the rotation of the island slows down.

Slightly before disruption, the intrinsic toroidal rotation starts to drop quite rapidly, departing from the variation of the island frequency. The reason for this behavior is not yet entirely clear to us. One possibility is the effect of inversion of the radial electric field, as observed in TUMAN-3M [31]. As it is well-known, the radial component of the ambipolar electric field plays a substantial role on plasma rotation and magnetic island evolution $[12,32]$. In the experiments carried out in TUMAN-3M, it was found that, as the amplitude of a peripheral $(|m / n|=3 / 1)$ island increased, the radial electric field reversed sign from negative (inward) to positive (outward), while the direction of island rotation remained unchanged. This result was explained by a theoretical model based upon the hypothesis that, as the island reaches a large size, 
the perturbed magnetic field in the peripheral plasma layer becomes stochastic, substantially increasing electron losses; then, the ambipolarity condition requires the radial electric field to reverse [33].

As mentioned before, in TCABR the plasma rotates toroidally in the direction of the toroidal magnetic field, opposite to the plasma current. This is consistent with a torque driven by an inward ion current crossed with the poloidal magnetic field, i.e., with an electric field pointing inward. However, as the main $|m / n|=2 / 1$ island saturates, the $m=3-5$ components of the perturbed magnetic field are observed for $t>$ $75 \mathrm{~ms}$. If we assume that these components give rise to a stochastic layer, we can expect radial electric field reversal, according to the model proposed by Kaveeva et al. [33], reducing the toroidal rotation, as observed experimentally. Therefore, although we have not measured the electric field directly in these experiments (in particular because the $|m / n|=2 / 1$ island is away from the plasma boundary), the decrease in the toroidal rotation velocity is at least in qualitative agreement with the theoretical model.

\section{CONCLUSIONS}

In conclusion, we have carried out a detailed experimental investigation of the behavior of the intrinsic toroidal rotation in disruptive discharges with large $|m / n|=2 / 1$ magnetic islands. The interplay between the magnetic island and the bulk plasma is such that the plasma intrinsic toroidal velocity increases as the island rotation decreases, indicating an "island-bulk plasma" interaction driven by the island. Measurements carried out with NBI mask this effect, as NBI adds significant momentum to both the plasma and the island, such that both practically acquire the same velocity. Furthermore, we have also shown that, somewhat before disruption, the intrinsic toroidal rotation decreases rapidly. This behavior was observed in all discharges of this experimental campaign, and we plan to investigate it in more detail. For this purpose, the rotation diagnostic is being further improved to reduce its uncertainty and increase its time resolution. The interplay between island growth and intrinsic toroidal rotation clearly indicates that the magnetic field perturbations affect the latter, and this seems to be an important effect to be taken into account in theoretical models to explain it.

\section{ACKNOWLEDGMENTS}

This work is honor of the memory of Anatolii Borisovich Mikhailovskii. He and his late disciple Vladimir Semyonovich Tsypin have played a fundamental role in stimulating the study of intrinsic plasma rotation and transport in the TCABR tokamak. Although Anatolii has never visited the University of São Paulo, he was always prompt to kindly help in the interpreta- tion and modeling of our experimental results. This work has been supported by different grants from FAPESP (Fundação de Amparo à Pesquisa do Estado de São Paulo), Project 2011/50773-0, and CNPq (Conselho Nacional de Desenvolvimento Científico e Tecnológico), Project 480733/2013-9, and by the University of São Paulo and University of Porto Collaboration Agreement.

\section{REFERENCES}

1. F. Salzedas, F. C. Schüller, A. A. M. Oomens, and RTP Team, Phys. Rev. Lett. 88, 075002 (2002).

2. P. C. de Vries, M. F. Johnson, B. Alper, P. Buratti, T. C. Hender, H. R. Koslowski, V. Riccardo, and JET-EFDA Contributors, Nucl. Fusion 51, 053018 (2011).

3. H. P. Furth, J. Killen, and M. N. Rosenbluth, Phys. Fluids 6, 459 (1963).

4. B. Coppi, Phys. Fluids 7, 1501 (1964).

5. P. H. Rutherford, Phys. Fluids 16, 1903 (1973).

6. R. B. White, D. A. Monticello, M. N. Rosenbluth, and B. V. Waddell, Phys. Fluids 20, 800 (1977).

7. B. D. Scott, A. B. Hassam, and J. F. Drake, Phys. Fluids 28, 275 (1985).

8. B. Lazzaro and M. F. F. Nave, Phys. Fluids 31, 1623 (1988).

9. M. F. F. Nave and J. A. Wesson, Nucl. Fusion 30, 2575 (1990).

10. R. Fitzpatrick, Nucl. Fusion 33, 1049 (1993).

11. J. H. F. Severo, I. C. Nascimento, V. S. Tsypin, and R. M. O. Galvão, Nucl. Fusion 43, 1047 (2003).

12. J. H. F. Severo, I. C. Nascimento, V. S. Tsypin, Y. K. Kuznetsov, E. A. Saettone, A. Vannucci, R. M. O. Galvão, M. Tendler, and A. B. Mikhailovskii, Phys. Plasmas 11, 846 (2004)

13. R. Fitzpatrick, Phys. Plasmas 5, 3325 (1998).

14. J. A. Snipes, D. J. Campbell, P. S. Haynes, T. C. Hender, M. Hugon, P. J. Lomas, N. J. Lopes Cardozo, M. F. F. Nave, and F. C. Schüller; Nucl. Fusion 28, 1085 (1988).

15. M. F. M. de Bock, I. G. J. Classen, C. Bush, R. J. E. Jaspers, H. R. Koslowski, B. Unterberg, and the TEXTOR Team, Nucl. Fusion 48, 015007 (2008).

16. J. S. de Grassie, Plasma Phys. Controlled Fusion 51, 124047 (1999).

17. B. Coppi, Nucl. Fusion 42, 1 (2002).

18. J. H. F. Severo, G. Ronchi, R. M. O. Galvão, I. C. Nascimento, Z. O. Guimarães-Filho, Yu. K. Kuznetsov, M. F. F. Nave, A. M. Oliveira, F. do Nascimento, and M. Tendler; Nucl. Fusion 55, 093001 (2015).

19. A. I. Smolyakov, A. Hirose, E. Lazzaro, G. B. Re, and J. D. Callen, Phys. Plasmas 2, 1581 (1995).

20. M. P. Alonso, A. C. A. Figueiredo, F. O. Borges, J. I. Elizondo, R. M. O. Galvão, J. H. F. Severo, O. C. Usuriaga, L. A. Berni, and M. Machida, Rev. Sci. Instrum. 81, 10D529 (2010).

21. M. Okamoto, S. Takamura, N. Ohno, S. Kajita, Y. Kikuchi, Y. Uesugi, T. Ozeki, Y. Kawano, and M. Sugihara, Nucl. Fusion 47, 1106 (2007). 
22. A. Bortolon, Y. Camenen, A. N. Karpushov, B. P. Duval, Y. Andrebe, L. Federspiel, O. Sauter, and the TCV Team, Nucl. Fusion 53, 023002 (2013).

23. R. E. Bell, R. Andre, S. M. Kaye, R. A. Kolesnikov, B. P. LeBlanc, G. Rewoldt, W. X. Wang, and S. A. Sabbagh, Phys. Plasmas 17, 082507 (2013).

24. R. C. Isler, Plasma Phys. Controlled Fusion 36, 171 (1994).

25. J. H. F. Severo, I. C. Nascimento, Yu. K. Kuznetsov, R. M. O. Galvão, Z. O. Guimarães-Filho, F. O. Borges, O. C. Usuriaga, J. I. Elizondo, W. P. de Sá, and E. K. Sanada, Nucl. Fusion 49, 115026 (2009).

26. Y. B. Kim, P. H. Diamondt, and R. J. Groebner, Phys. Fluids B3, 2050 (1991).

27. I. Semenov, S. Mirnov, I. C. Nascimento, R. M. O. Galvão, Yu. Kuznetsov, A. Belov, and A. Vannucci, Rev. Sci. Instrum. 70, 867 (2005).
28. V. A. Kornev, L. G. Askinazi, M. I. Vil'dzhyunas, V. E. Golant, N. A. Zhubr, S. V. Krikunov, S. V. Lebedev, V. V. Rozhdestvenski, and A. S. Tukachinski, Plasma Phys. Rep. 31, 803 (2005).

29. Y. Z. Zhang, R. Denton, S. Mahajan, C. Jiayu, and A. Wootton, Phys. Rev. Lett. 65, 2877 (1990).

30. A. M. M. Fonseca, V. S. Tsypin, R. M. O. Galvão, Y. K. Kuznetsov, I. C. Nascimento, R. P. da Silva, E. A. Saettone, and A. Vannucci, Phys. Plasmas 12, 052501 (2005)

31. L. G. Askinazi, V. E. Golant, V. A. Kornev, S. V. Lebedev, A. S. Tukachinsky, M. I. Vildjunas, and N. A. Zhubr, Plasma Phys. Controlled Fusion 48, A85 (2006).

32. A. B. Mikhailovskii, V. D. Pustovitov, V. S. Tsypin, and A. I. Smolyakov, Phys. Plasmas 7, 1204 (2000).

33. E. Kaveeva, V. Rozhansky, and M. Tendler, Nucl. Fusion 48, 075003 (2008). 